\title{
DATA DRIVEN CHOICE OF THRESHOLD IN CEPSTRUM BASED SPECTRUM ESTIMATE
}

\author{
M.Venakatanarayana ${ }^{1}$, Dr.T.Jayachandra Prasad ${ }^{2}$ \\ ${ }^{1}$ Department of ECE, KSRMCE, Kadapa, Andhra Pradesh, India \\ narayanamoramegmail.com \\ ${ }^{2}$ RGMCET, Nandyal, Kurnool, Andhra Pradesh, India \\ jp.talari@gmail.com
}

\begin{abstract}
The technique of cepstrum thresholding, which is shown to be an effective, yet simple, way of obtaining a smoothed non parametric spectrum estimate of a stationary signal. The major problem of this method is the choice of the threshold value for variance reduction of spectrum estimates. This paper proposes a new threshold selection method which is based on cross validation schemes such as Leave-One-Out, LeaveTwo-Out and Leave-Half-Out. This new methods are easy to describe, simple to implement, and does not impose severe conditions on the unknown spectrum. Numerical results suggest that this new methods are shown to be in agreement with those obtained when the spectrum is fully known.
\end{abstract}

\section{KEYWORDS}

Cross Validation, Cepstrum, Periodogram, Integrated Mean Square Error, Unbiased and Smoothing.

\section{INTRODUCTION}

The cepstrum is a guide to signal processing in literature [1], [2]. Since, it is applied widely in various fields such as speech processing, image processing, filter design, seismology and geology etc., [3], [4], [5]. As mentioned in [3] and [6], given the many successful stories of applications of cepstrum, it is certain that new and useful applications of cepstrum come in future. Hence, the goal is to propose new methods, which have high performance procedures for cepstral estimation. Consider a stationary, discrete-time, real valued signal $x(t), t=0,1,2, \ldots \ldots$. , with covariance sequence $\left\{r_{k}\right\}_{k=-\infty}^{\infty}$ and power spectral density (or spectrum) $\phi_{p}(\omega)$ where $\omega \in[-\pi, \pi]$. The idea in this paper is to estimate the spectrum $\phi(\omega)$ from a set of observed samples $\{x(t)\}_{t=0}^{N-1}$ of the signal.

The periodogram estimate of $\phi(\omega)$ is given by [7-9]

$$
\hat{\phi}_{p}(\omega)=\frac{1}{N}\left|\sum_{t=0}^{N-1} x(t) e^{-i \omega t}\right|^{2}
$$

where the subscript 'p' denotes the Periodogram estimate. $\hat{\phi}_{p}(\omega)$ can also be written in terms of the covariance sequence as 


$$
\hat{\phi}_{p}(\omega)=\sum_{k=-(N-1)}^{N-1} \hat{r}_{k} e^{-i \omega k}
$$

where $\hat{r}_{k}$ denotes the following estimate of $\mathrm{r}^{k}$

$$
\hat{r}_{k}=\frac{1}{N} \sum_{t=k}^{N-1} x(t) x(t-k)
$$

where $k=0,1, \ldots \ldots \ldots . . . N-1, \quad \hat{r}_{-k}=\hat{r}_{k}$

$$
\text { Let } \quad \omega_{l}=\frac{2 \pi}{N} l, l=0, \ldots \ldots . ., N-1
$$

denote the Fourier grid of the angular frequency axis. $\hat{\phi}_{p}(\omega)$ can be computed efficiently by means of a Fast Fourier transform (FFT) algorithm.

The periodogram estimate is an asymptotically unbiased but inconsistent estimate of the true spectrum [7-9]. The variance of periodogram estimate is high; it does not converge to zero as $N$ increases, but approach to $\Phi^{2}\left(\omega_{l}\right)$. To solve the above problem, many periodogram smoothing techniques (in both the time, lag and frequency domain) have been proposed. All these techniques suffer from the drawback of having to carefully select the window and the span, for which there are no clear-cut guidelines. Data-dependent choices of the window span are hard to make, due to the complicated statistical properties of the covariance estimates. In recent days, the periodogram estimates via cepstrum thresholding [6], [10], called SThresh [17] have very simple statistical properties. The choice of threshold level is almost automatic way, since the threshold level is selected manually by a procedure for which there are clear guidelines and for which only minor prior information is needed.

\section{RELATED WORK}

There are several approximately fully automatic schemes are available to select the threshold. These include namely Uniformly Most Power Unbiased Test (UMPUT) [10], the Bayesian Information Criterion (BIC) [10] and The Kolmogorov Structure Function (KSF) [11]. All these are useful whenever true power spectrum is known to fix the optimum threshold. But in practice, the true spectrum is unknown. To overcome this attempt has been made to develop fully automated threshold selection method such as Leave-One-Out Cross Validation (LOOCV) in the absence of the knowledge of true spectrum [16]. We extended the Cross Validations Schemes such as Leave-Two-Out and Leave-Half-Out to select the threshold in non parametric smoothed power spectrum estimation via Cepstrum thresholding.

The paper is organized and is as follows. In the next section the cepstrum based smoothing technique is introduced. In section 4 the Cross Validation schemes for choosing the optimum threshold are described and in section 5 some numerical examples have been presented to illustrate the benefit of the proposed algorithm. Comparison among the various threshold selection schemes is presented in section 6 . 
Signal \& Image Processing : An International Journal (SIPIJ) Vol.2, No.4, December 2011

\section{Smoothed Spectral Estimation via Cepstrum Thresholding - STHRESH}

The Cepstral coefficients are defined as

$$
c_{k}=\frac{1}{N} \sum_{l=0}^{N-1} \ln \left[\phi\left(\omega_{l}\right)\right] e^{j \omega_{l} k}, k=0,1, \ldots \ldots \ldots . . . N-1
$$

where it is assumed that $\phi\left(\omega_{l}\right)>0, \forall l$. The cepstral coefficients have several interesting features, one of which is mirror symmetry:

$$
c_{N-k}=c_{k}, \quad k=0,1, \ldots \ldots \ldots, N / 2
$$

which mean that only half of the sequence $c_{0}, \ldots \ldots \ldots . ., c_{N / 2}$, is distinct. The other half is obtained from $c_{1}, \ldots \ldots \ldots . . ., c_{(N / 2)-1}$, via $(6)$.

Using the periodogram estimate in (1), a common estimate of the cepstral coefficients is obtained by replacing $\phi(\omega)$ in (5) with $\hat{\phi}_{p}(\omega)$, which given [6], [7].

$$
\begin{aligned}
\hat{c}_{k} & =\frac{1}{N} \sum_{l=0}^{N-1} \ln \left[\hat{\phi}_{p}\left(\omega_{l}\right)\right] e^{j \omega_{l} k}+\gamma \delta_{k, 0}, \\
k & =0, \ldots \ldots \ldots \ldots . . M, \\
\delta_{k, 0} & =\left\{\begin{array}{cc}
1 & \text { if } k=0 \\
0 & \text { else }
\end{array}\right.
\end{aligned}
$$

$\mathrm{M}=\mathrm{N} / 2$ and $\gamma=0.577216 \ldots$ (the Euler's constant).

It can be shown that in large samples, the estimated cepstral coefficients $\left\{\hat{c}_{k}\right\}_{k=0}^{M}$ are independent normally distributed random variables [18]:

$$
\begin{gathered}
\hat{c}_{k} \sim N\left(c_{k}, s_{k}^{2}\right) \\
s_{k}^{2}=\left\{\begin{array}{lc}
\frac{\pi^{2}}{3 N} & \text { if } k=0, \ldots ., M \\
\frac{\pi^{2}}{6 N} & \text { if } k=1, \ldots \ldots . ., M-1
\end{array}\right.
\end{gathered}
$$

with the above equations in mind, the idea behind cepstrum thresholding is taken from the literature [23-25]. Let $\tilde{c}_{k}$ be a new estimate of $c_{k}$ and note that $\tilde{c}_{k}=0$ has a mean squared error (MSE) equal to $c_{k}^{2}$. This estimate is preferred to $\hat{c}_{k}$ as long as $c_{k}^{2} \leq s_{k}^{2}$. Now let 
Signal \& Image Processing : An International Journal (SIPIJ) Vol.2, No.4, December 2011

$$
S=\left\{k \in[0, M] \mid c_{k}^{2} \leq s_{k}^{2}\right\}
$$

and let $\tilde{S}$ be an estimate of the set $\mathrm{S}$. Thresholding $\left\{\hat{c}_{k}\right\}_{k \in \tilde{S}}$ gives the following new estimates of $c_{k}$ :

$$
\tilde{c}_{k}=\left\{\begin{array}{cc}
0 & \text { if } k \in \tilde{S} \\
\hat{c}_{k} & \text { else }
\end{array} \quad k=0, \ldots \ldots, M\right.
$$

A good estimate of $\mathrm{S}$ is given by

$$
\tilde{S}=\left\{k \in[0, M]\left|\hat{c}_{k}\right| \leq \mu s_{k}\right\}
$$

where the parameter $\mu$ controls the risk of concluding that $\left|c_{k}\right|$ is "significant" when this is not true, the so called "false alarm probability". There are three different methods to set the threshold level $\mu$, they are outlined below.

UMPUT [10] - The expression is derived by combining a uniformly most powerful unbiased test with some empirical evidence and it is given by [20]

For $N \in(128,2048)$ :

$$
\mu=\mu_{0}+\frac{N-128}{1920}
$$

where

$$
\mu_{0}=\left\{\begin{array}{cr}
4 & \text { for a broadband signal with small dynamic range } \\
3 & \text { for a broadband signal with } l \arg \text { e dynamic range } \\
2 & \text { for a narrowband signal with very } l \arg \text { e dynamic range }
\end{array}\right.
$$

BIC [10] - In this case, the choice of the threshold depends on the Bayesian Information Criterion [19]. The formula of $\mu_{\text {is: }}$

$$
\mu_{B I C}=1+(\ln M)^{1 / 2}, \quad \text { where } \mathrm{M}=\mathrm{N} / 2
$$

KSF [11] - The value of $\mu$ is selected such that to minimize the Kolmogorov structure function (KSF) as it is formulated in [21]. The following threshold is used in cepstral nulling

$$
\mu_{K S F}=1+l^{*} \text {, where } l^{*}=\arg \min _{l} h_{c}(l) \text {, the KSF is denoted by } h_{c}(l)
$$

This means that $\mu$ belongs to the interval $\left(\mu_{0}, \mu_{0}+1\right)$. For other intervals of the sample length, $\mathrm{N}$, similar rules can be developed. The smoothed spectral estimate corresponding to $\left\{\tilde{c}_{k}\right\}$ is given by: 


$$
\tilde{\phi}_{c e p}\left(\omega_{l}\right)=\exp \left[\sum_{k=0}^{N-1} \tilde{c}_{k} e^{-i \omega_{l} k}\right], l=0, \ldots \ldots, N-1,
$$

where the subscript cep signifies its cepstrum dependence. The final scaled spectrum estimate $\hat{\phi}_{c e p}\left(\omega_{l}\right)$ is then given by

$$
\begin{gathered}
\hat{\phi}_{c e p}\left(\omega_{l}\right)=\hat{\alpha} \tilde{\phi}_{c e p}\left(\omega_{l}\right), l=0, \ldots \ldots . ., N-1 \\
\hat{\alpha}=\frac{\sum_{l=0}^{N-1} \hat{\phi}_{p}\left(\omega_{l}\right) \tilde{\phi}_{c e p}\left(\omega_{l}\right)}{\sum_{l=0}^{N-1} \tilde{\phi}_{c e p}^{2}\left(\omega_{l}\right)}
\end{gathered}
$$

The above outlined smoothing scheme was called SThresh. We now proceed to automate the selection of $\mu$, using a cross-validation scheme.

\section{Cross-Validation Based Threshold Selection (CV-SThreSh)}

The idea of using cross-validation for selection of smoothing parameters is quite appealing. The selection of the bandwidth of estimates that are based on a discrete periodogram average, by means of cross-validation, is discussed in [12-14]. The concept of cross validation is applied to find an optimum value of the threshold $\mu$. This optimal threshold value will be used to smooth the spectrum with the procedure outlined in section 3. First various types of cross validation methods are discussed[15], then the concept of Leave-One-Out Cross Validation(LOOCV) is explored to find an optimum threshold value[16].

\subsection{Definitions}

Stein's Unbiased Risk Estimation (SURE) - Choose the value of threshold $\mu$ at which the integrated mean square error (IMSE) is minimum. The IMSE of $\left\{\tilde{\Phi}_{k}\right\}$ is given by

$$
\rho=\sum_{k=0}^{N-1} E\left(\tilde{\Phi}_{k}-\Phi_{k}\right)^{2}
$$

K-Fold Cross-Validation-In k-fold cross-validation the data is first partitioned into k equally (or nearly equally) sized segments or folds. Subsequently k iterations of training and validation are performed such that within each iteration a different fold of the data is held-out for validation while the remaining k- 1 folds are used for learning. Data is commonly stratified prior to being split into $\mathrm{k}$ folds. Stratification is the process of rearranging the data as to ensure each fold is a good representative of the whole. For example in a binary classification problem where each class comprises $50 \%$ of the data, it is best to arrange the data such that in every fold, each class comprises around half the instances.

Leave-One-Out Cross-Validation (LOOCV) -Leave-one-out cross-validation is a special case of $\mathrm{k}$-fold cross-validation where $\mathrm{k}$ equals the number of instances in the data. In other words, all the data except for a single observation are used for training and the model is tested on that single observation. 
Signal \& Image Processing : An International Journal (SIPIJ) Vol.2, No.4, December 2011

Leave-Two-Out Cross-Validation (LTCOCV) -In this case, almost all the data except for two observations are used for training the model and the model is tested on the left out two observations.

Leave-Half-Out Cross-Validation (LHCOCV)-In this case, the data is divided into two equal segments. One segment is used for training the model and other one for testing the model. Leavehalf-out cross validation is simple to implement, and achieves same performance as that of the above techniques with the reduced computational complexity.

\subsection{Periodogram Smoothing via Cross Validation}

If we knew the true underlying spectrum $\phi(\omega)$, we would find $\mu$ by minimizing the integrated mean squared error (IMSE) of $\hat{\phi}_{\text {cep }}$ with respect to $\mu$, where

$$
\operatorname{IMSE}\left(\hat{\phi}_{\text {cep }}\right)=\frac{1}{N} \sum_{j=0}^{N-1} E\left[\hat{\phi}_{c e p}\left(\omega_{j}\right)-\phi\left(\omega_{j}\right)\right]^{2}
$$

and $E[$.$] denotes the expectation. Unfortunately, in most practical situations, \phi(\omega)$ is unknown and computing the IMSE is therefore not possible. However, cross-validation schemes can be utilized to find an estimate of IMSE, for instance, the following estimate is given by

$$
C V \operatorname{MSE}\left(\hat{\phi}_{c e p}\right)=\frac{1}{N} \sum_{j=0}^{N-1}\left[\hat{\phi}_{c e p}^{-j}\left(\omega_{j}\right)-\hat{\phi}_{p}\left(\omega_{j}\right)\right]^{2}
$$

here, $\hat{\phi}_{c e p}{ }^{-j}\left(\omega_{j}\right)$ is the leave-one-out version of $\hat{\phi}_{c e p}\left(\omega_{j}\right)$, constructed such that $\hat{\phi}_{c e p}^{-j}\left(\omega_{j}\right)$ is independent of $\hat{\phi}_{p}\left(\omega_{j}\right)$ for all $\mathrm{j}$. This is achieved by first calculating

$$
\hat{c}_{k}^{-j}=\frac{1}{N} \sum_{\substack{l=0 \\ l \neq j}}^{N-1} \ln \left[\hat{\phi}_{p}\left(\omega_{l}\right)\right] e^{j \omega_{l} k}
$$

This leave-one-out estimate can be efficiently obtained from

$$
\hat{c}_{k}^{-j}=\hat{c}_{k}-\frac{1}{N} \ln \left[\hat{\phi}_{P}\left(\omega_{j}\right)\right] e^{i \omega_{l} k}
$$

The cepstral coefficients are then thresholded according to (12) for a particular choice of $\mu$. Finally, the leave-one-cut estimate in (23) is given by

$$
\hat{\phi}_{c e p}^{-j}\left(\omega_{j}\right)=\hat{\alpha} \exp \left[\sum_{k=0}^{N-1} \tilde{c}_{k}^{-j} e^{-i \omega_{j} k}\right] \quad j=0, \ldots \ldots \ldots ., N-1
$$

where $\tilde{c}_{k}^{-j}$ denotes the threshold version of $\hat{c}_{k}^{-j}$ and $\hat{\alpha}$ is obtained by replacing $\tilde{\phi}_{c e p}(\omega)$ with its leave-one-out estimate $\tilde{\phi}_{c e p}{ }^{-j}(\omega)$ in (21). The optimal thresholding parameter $\mu$ is then the one that minimizes the criterion (23). 
Signal \& Image Processing : An International Journal (SIPIJ) Vol.2, No.4, December 2011

The Algorithm steps for proposed smoothing scheme CV-SThresh to find the optimal threshold parameter, $\mu_{\text {opt }}$.

1. From $x(t), t=0,1, \ldots \ldots, N-1$, compute $\hat{\phi}_{p}(\omega)$ and $\hat{c}_{k}$.

2. Choose a $\mu \in\left[0, \mu_{\max }\right]$. Empirical studies have shown that taking $\mu_{\max }=10$ is a good general choice.

3. Find an estimate of $\operatorname{IMSE}\left(\hat{\phi}_{\text {cep }}(\omega)\right)$ by efficiently evaluating $\operatorname{CVMSE}\left(\hat{\phi}_{\text {cep }}(\omega)\right)$ in $(23)$. The efficient way of obtaining $\hat{c}_{k}^{-j}$ in (25) reduces computation by a factor of $\log N$.

4. Repeat the above step over the range $\left[0, \mu_{\max }\right]$.

5. Find the value of $\mu$ that minimizes $C \operatorname{VMSE}\left(\hat{\phi}_{\text {cep }}(\omega)\right)$.

6. Finally compute the smoothed spectrum as done in section 3, using $\mu_{\text {opt }}$ as a threshold.

\section{SimUlation}

The performance of the proposed CV-SThresh method is illustrated by using the broadband and narrowband signals and also for MST radar data.

\subsection{Broadband Signal}

Consider a broadband, second order MA process. The signal $x(t)$ was generated using the moving average equation

$$
x(t)=e(t)+0.55 e(t-1)+0.15 e(t-2), \quad t=0, \ldots \ldots \ldots, N-1
$$

where $e(t)$ is a zero mean, unit variance normal white noise. We generated 100 realizations, each of length $\mathrm{N}=512$ of the process. In Fig. 1 we show the true spectrum, periodogram based spectrum and the smoothed spectrum via CV-SThresh. The variance and mean square error parameters of these methods are shown in Fig.2. We clearly see that the variance of the smoothed spectrum via $\mathrm{CV}$-SThresh is significantly smaller than that of the peridogram. The optimal $\mu$ can be obtained from Fig.4 (a) by finding the $\mu$ corresponding to the minimum value of IMSE. It is clearly seen from Fig. 3 that the true and the CV estimate of IMSE have a minimum at almost the same point. Cross- validation therefore gives a nearly optimal value of $\mu$ for thresholding. 
Signal \& Image Processing : An International Journal (SIPIJ) Vol.2, No.4, December 2011

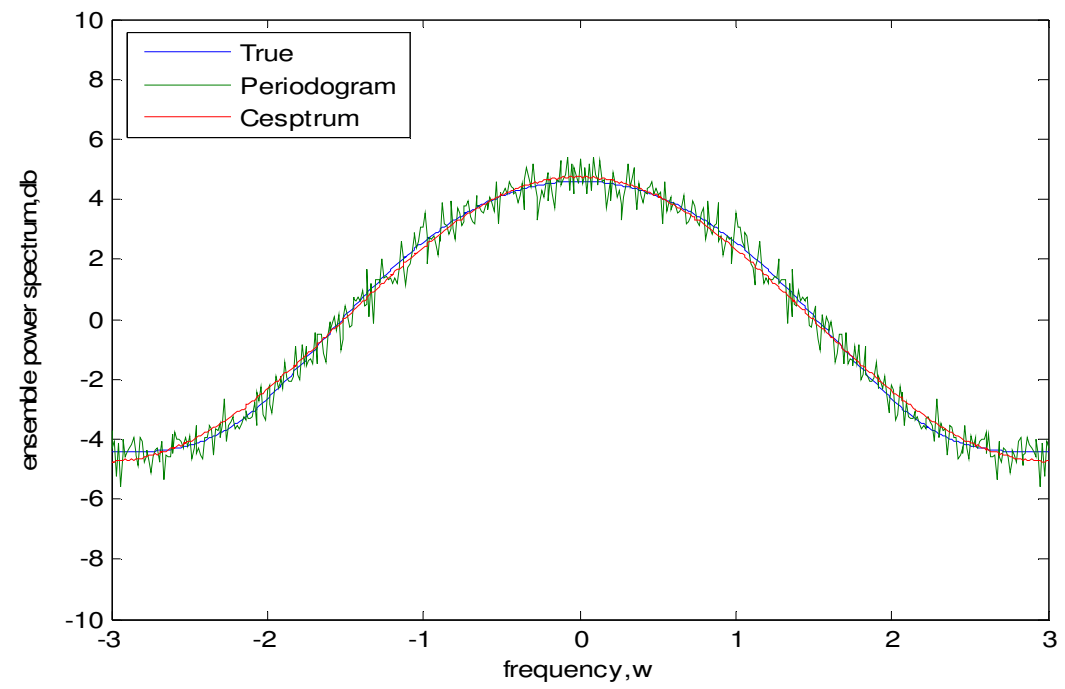

Figure 1. True spectrum $\phi(\omega)$, the periodogram estimate $\hat{\phi}_{p}(\omega)$ and the cepstrum based spectrum estimate $\hat{\phi}_{\text {cep }}(\omega)$ versus frequency $\omega \mathrm{rad} / \mathrm{s}$, for a broadband MA signal $\mathrm{N}=512$
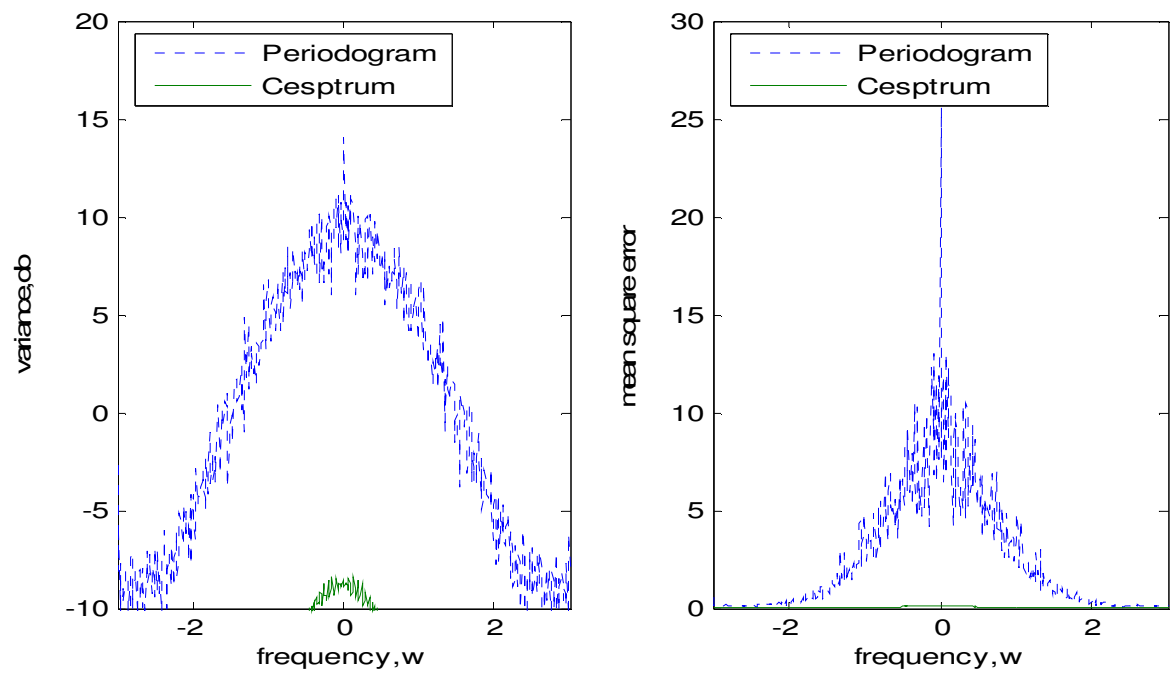

Figure 2. Variance and Mean square error of $\hat{\phi}_{p}(\omega)$ and $\hat{\phi}_{\text {cep }}(\omega)$ versus frequency $\omega$ 
Signal \& Image Processing : An International Journal (SIPIJ) Vol.2, No.4, December 2011

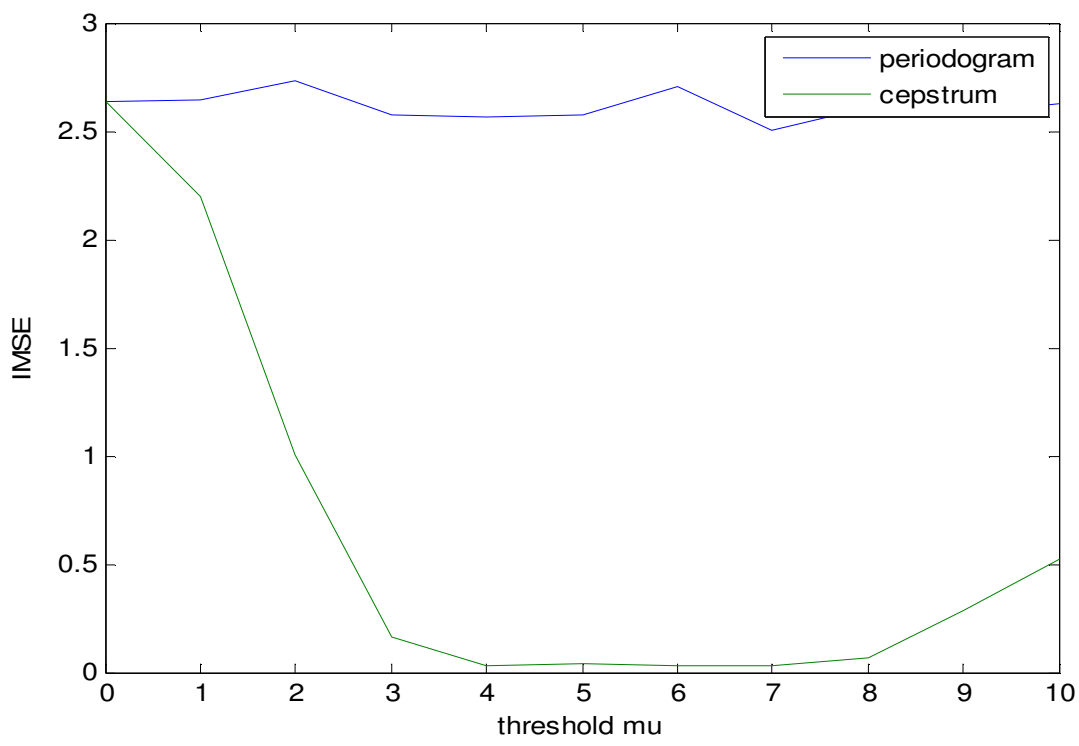

Figure 3. IMSE $\left(\hat{\phi}_{p}(\omega)\right)$ and IMSE $\left(\hat{\phi}_{c e p}(\omega)\right)$ versus the threshold $\mu$

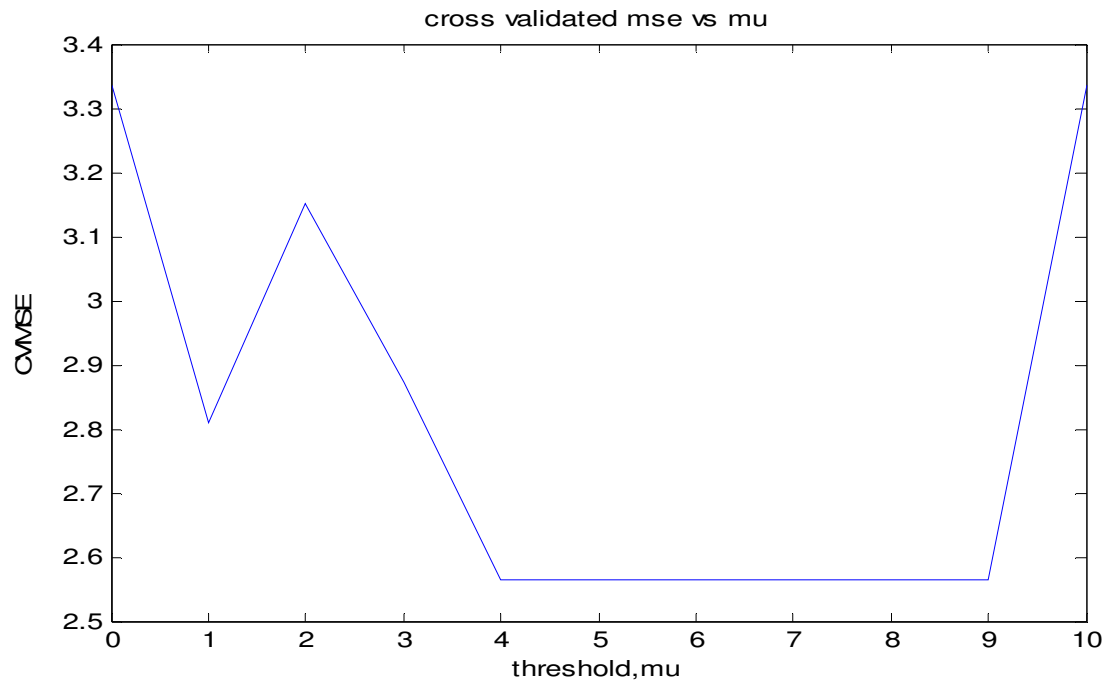

Figure 4. CVMSE $\left(\hat{\phi}_{\text {cep }}(\omega)\right)$ versus the threshold $\mu$ using cross validation method.

\subsection{Narrowband Signal}

Consider a narrowband ARMA process represented by

$$
\begin{aligned}
& x(t)-1.6408+2.2044 x(t-1) x(t-2)-1.4808 x(t-3)+ \\
& 0.8145 x(t-4)=e(t)+1.5857 e(t-1)+0.9604 e(t-2), \quad t=0,1, \ldots . N-1
\end{aligned}
$$

where $e(t)$ is again a zero mean, unit variance normal white noise. We generated 1000 MonteCarlo simulations, each of length $\mathrm{N}=512$. as described in the broadband example, the optimal threshold parameter $\mu_{\text {opt }}$ has been obtained as the threshold that yields the minimum cross- 
Signal \& Image Processing : An International Journal (SIPIJ) Vol.2, No.4, December 2011

valedictory estimate of IMSE. For comparison, we have plotted the true spectrum together with the periodogram based spectrum and smoothed spectrum via CV-SThresh in Fig.5. The variance and mean square error parameters of these methods are shown in Fig.6. Fig.7 and 8 shows the IMSE and CVMSE curves versus $\mu$, used to find $\mu_{\text {opt }}$. In comparison, we see that the variance has been reduced but a bias has been introduced. When smoothing the spectrum via cepstrum thresholding, some of the energy is lost due to the truncation of $\hat{c}_{k}$ to zero in (12). For broadband signals, very few of the cepstral estimates are truncated so only a small bias is introduced, whereas for narrowband signals, many more coefficients are set to zero, thus causing the bias seen in Fig.5.

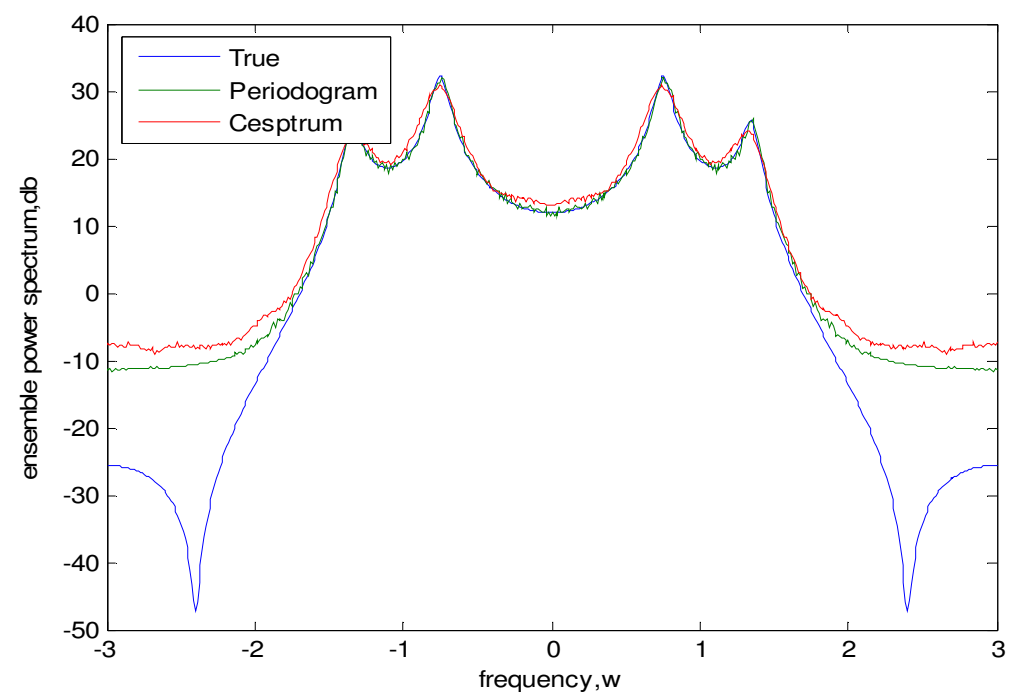

Figure 5. True spectrum $\phi(\omega)$, the periodogram estimate $\hat{\phi}_{p}(\omega)$ and the spectrum estimate $\hat{\phi}_{\text {cep }}(\omega)$ versus frequency $\omega \mathrm{rad} / \mathrm{s}$, for a narrowband ARMA signal $\mathrm{N}=512$
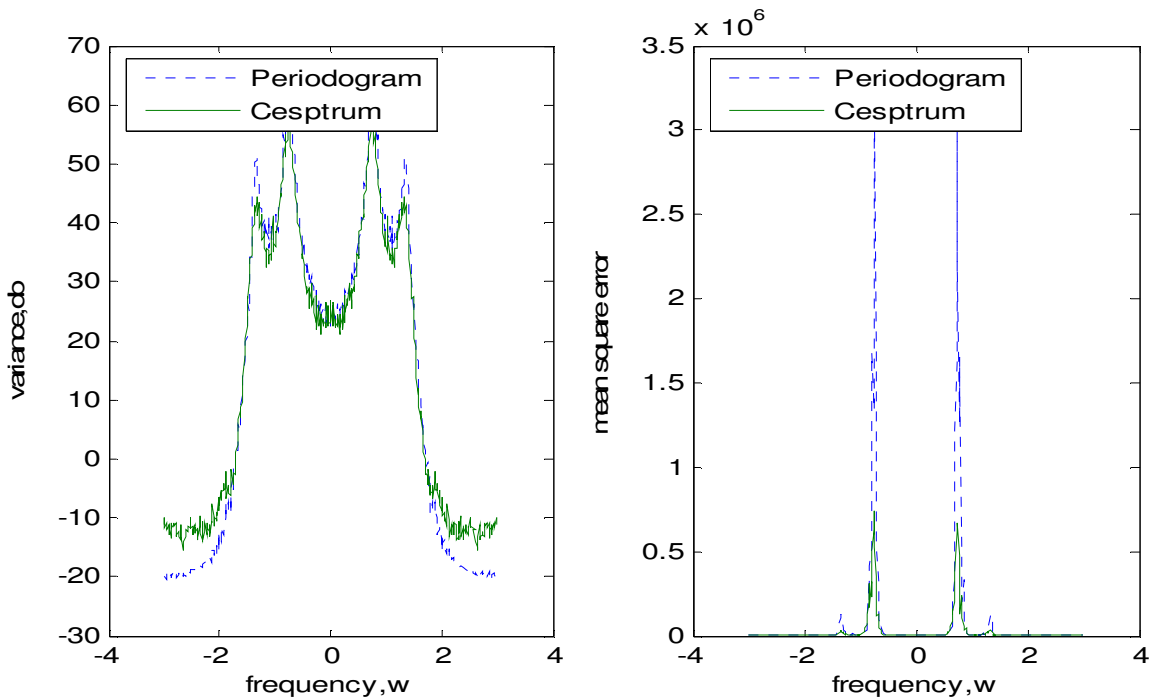

Figure 4. Variance and Mean square error of $\hat{\phi}_{p}(\omega)$ and $\hat{\phi}_{c e p}(\omega)$ versus frequency $\omega$ 
Signal \& Image Processing : An International Journal (SIPIJ) Vol.2, No.4, December 2011

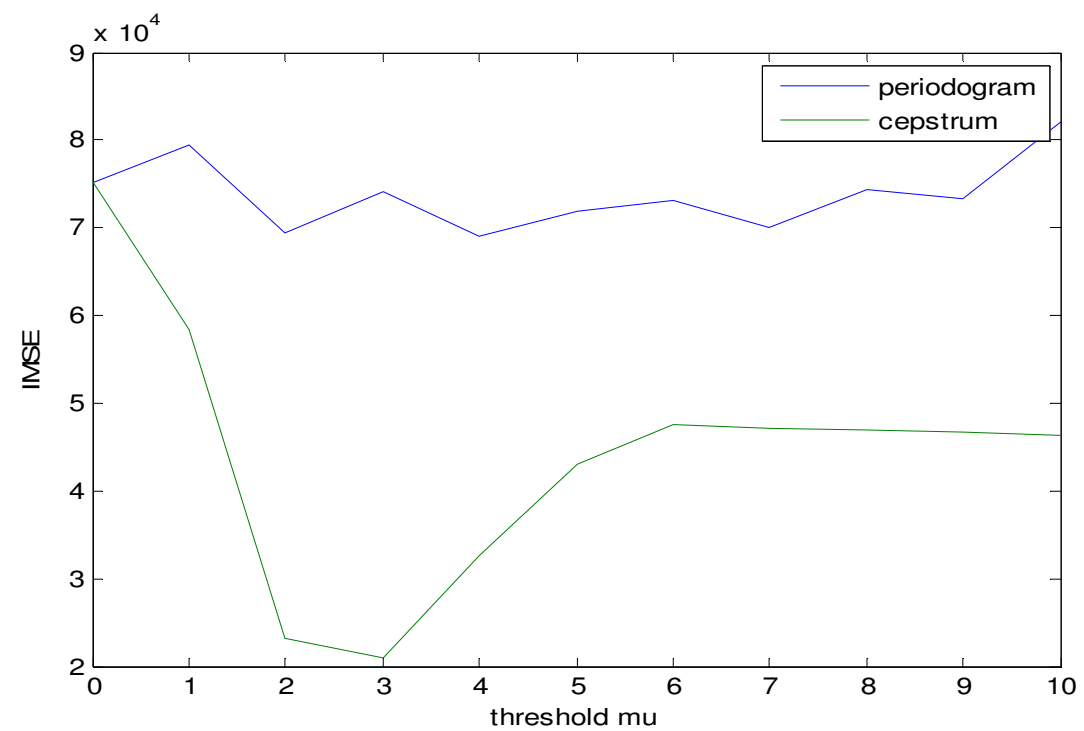

Figure 5. IMSE $\left(\hat{\phi}_{p}(\omega)\right)$ and IMSE $\left(\hat{\phi}_{c e p}(\omega)\right)$ versus the threshold $\mu$

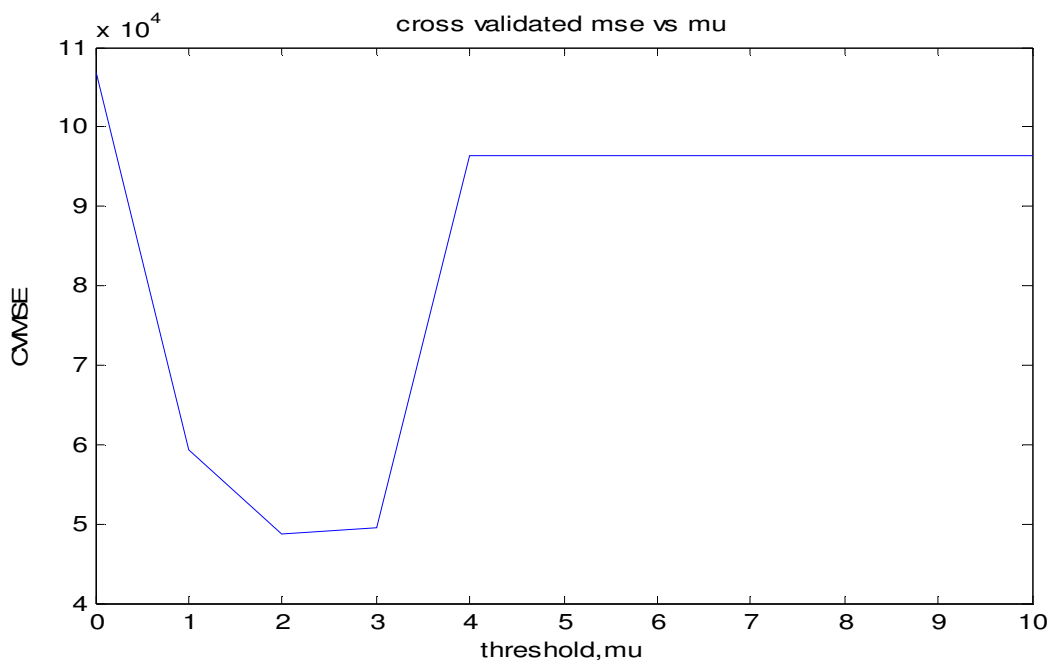

Figure 6. CVMSE ( $\hat{\phi}_{\text {cep }}(\omega)$ ) versus the threshold $\mu$ using cross validation method.

\subsection{MST Radar signal}

The MST radar data has been considered to estimate the spectrum based on the above described method. The MST atmospheric data is collected from the MST Radar centre at Gadhanki, Tirupati, India. The data set consists of 150 member functions, each having 256 samples. The proposed Cross Validation based method has been applied to radar data, it is observed that the spectrum of radar data is smoothed, which is shown in Fig.9. It is observed the spectrum estimated for MST radar data based on the proposed method is smoother than the spectrum estimated by periodogram estimate. 
Signal \& Image Processing : An International Journal (SIPIJ) Vol.2, No.4, December 2011

\section{COMPARISON AMONG SURE TECHNIQUE AND CROSS VALIDATION METHODS}

The Stein's unbiased risk estimation (SURE) technique and the cross validation schemes such as Leave-one-out (LOO), Leave two out (LTO) and Leave half out (LHO) methods have been applied to a broadband signal, a narrowband signal and a MST radar data for optimum threshold selection. Table.1 suggest that Leave half out cross validation is easy to implement, and achieves threshold value in agreement with other techniques with few computations.

TABLE.1. Threshold values of SURE, LOOCV, LTOCV and LHOCV

\begin{tabular}{|l|c|c|c|c|}
\hline \multirow{2}{*}{ Type of signal } & \multicolumn{4}{|c|}{ Optimum threshold value $\left(\mu_{0 p}\right)$} \\
\cline { 2 - 5 } & SURE Technique & LOOCV & LTOCV & LHOCV \\
\hline Broadband MA signal & 4 & 4 & 5 & 4 \\
\hline $\begin{array}{l}\text { Narrowband ARMA } \\
\text { signal }\end{array}$ & 3 & 3 & 2 & 2 \\
\hline MST Radar data & $*$ & 3 & 3 & 2 \\
\hline
\end{tabular}

* Not applicable duo to lack of known spectrum

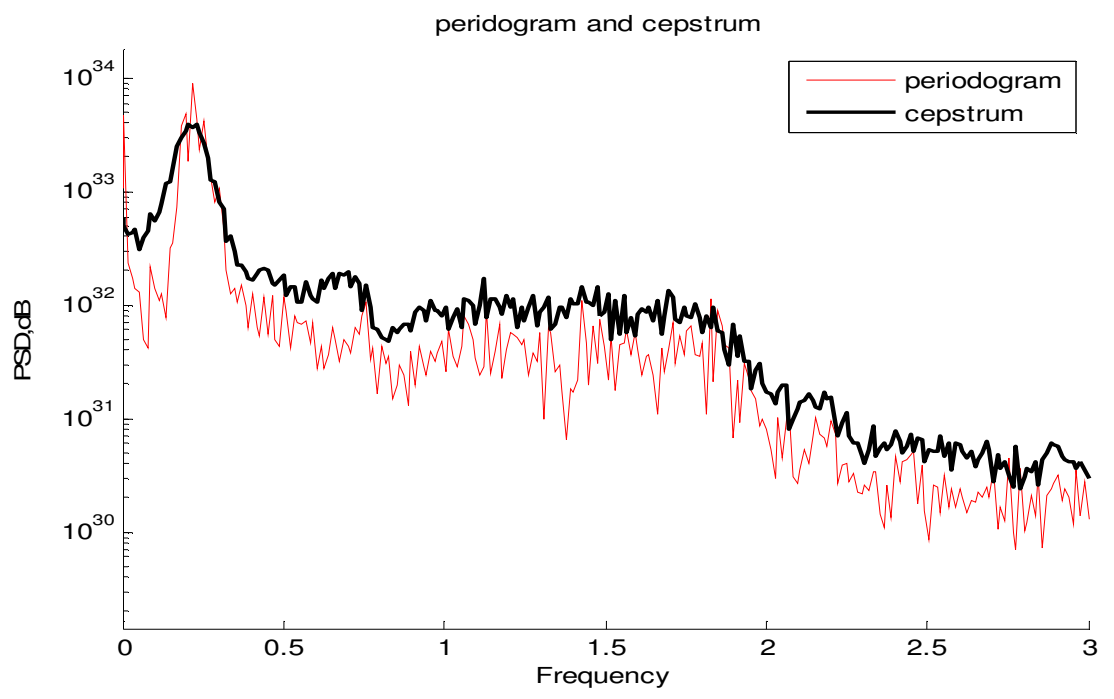

Figure 7. The periodogram estimate $\hat{\phi}_{p}(\omega)$ and the spectrum estimate $\hat{\phi}_{c e p}(\omega)$ versus frequency $\omega$ $\mathrm{rad} / \mathrm{s}$, for a radar data $\mathrm{N}=256$. 
Signal \& Image Processing : An International Journal (SIPIJ) Vol.2, No.4, December 2011

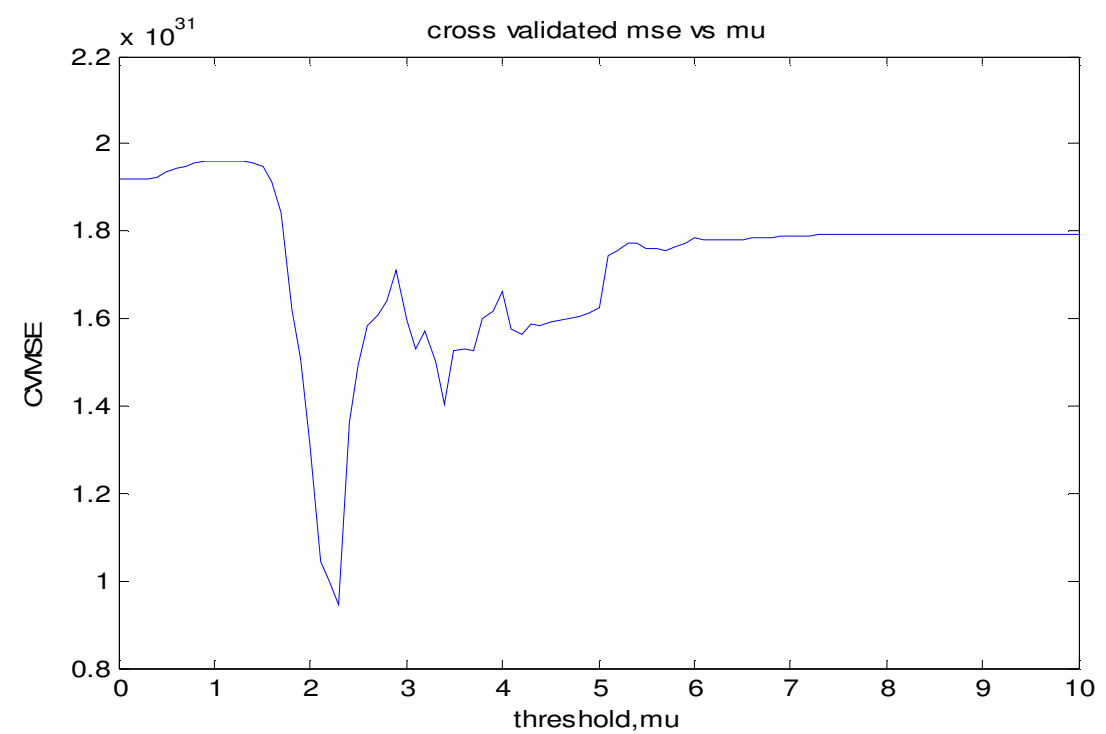

Figure 8. CVMSE ( $\hat{\phi}_{\text {cep }}(\omega)$ ) versus the threshold $\mu$ using cross validation method.

\section{Conclusions}

In this paper the CV-SThresh has been proposed, which is a new data-driven method for threshold selection in smoothed non-parametric spectral estimation. The technique of crossvalidation has been applied to a broadband, a narrowband and a MST radar data for determining the smoothed spectrums by automatically selecting optimum threshold value. The criterion used in this paper is a cross validatory estimate of the minimum mean square error. Three cross validation schemes: Leave-one-out, Leave-two-out and Leave-half-out are employed to select the optimum threshold level for the given $N$ Periodogram ordinates $\left(\hat{\Phi}_{P}\left(\omega_{k}\right)\right)$. The results obtained are in conformity with the existing results in [6], [16], derived using some apriori knowledge about the true spectrum. We observed that Leave-half-out Cross Validation scheme is simple to implement than the other methods, because it produces the good results with few computations.

\section{REFERENCES}

[1] D. G. Childers, D. P. Skinner, and R. C. Kemerait, "The cepstrum: A guide to processing," Proc. IEEE, vol. 65, no. 10, pp. 1428-1443, Oct. 1977.

[2] B. P. Bogert, M. J. R. Healy, and J. W. Tukey, "The frequency analysis of time series for echoes: Cepstrum, pseudo-autocovariance, cross-cepstrum and saphe cracking," in Time Series Analysis, M. Rosenblatt, Ed., 1963, pp. 209-243, ch. 15.

[3] A. V. Oppenheim and R. W. Schafer, "From frequency to quefrency: A history of the cepstrum," IEEE Signal Process. Mag., vol. 21, no. 5, pp. 95-106, Sep. 2004.

[4] L. R. Rabiner and R. W. Schafer, Digital Processing of Speech Signals. Englewood Cliffs, NJ: Prentice-Hall, 1978.

[5] V. Solo, "Modelling of two-dimensional random fields by parametric cepstrum," IEEE Trans. Inf. Theory, vol. IT-32, no. 6, pp. 743-750, Nov. 1986.

[6] P. Stoica and N. Sandgren, "Smoothed nonparametric spectral estimation via cepstrum Thresholding,” IEEE Signal Process. Mag., vol. 23, no. 6, pp. 34-45, Nov. 2006. 
Signal \& Image Processing : An International Journal (SIPIJ) Vol.2, No.4, December 2011

[7] P. Stoica and R.Moses, Spectral Analysis of Signals, Englewood Cliffs, NJ: Prentice Hall, 2005.

[8] D.B.Percival and A.T.Walden, Spectral Analysis for Physical Applications, Cambridge University Press, Cambridge, UK, 1993.

[9] M.B.Priestley, "Spectral Analysis and Time series" Volume-1, Academic Press, 1981.

[10] P.Stoica and N.Sandgren, "Total variance reduction via thresholding: Application to Cepstral Analysis”, IEEE transactions on Signal Processing, vol.55, no.1, pp.66-72, January 2007.

[11] C.D.Giurcaneanu and S.A.Razavi, "On the use of Kolmogorov structure function for periodogram smoothing", in Proceeding of the IEEE International Conference On Acoustics, Speech and Signal Processing, Dallas, Texas, USA, 2010, pp.3966-3969.

[12] C.M.Hurvich, "Data-Driven Choice of a Spectrum Estimate: Extending the Applicability of CrossValidation Methods", Journal of the American Statistical Association, vol.80, no.392, pp.933-940, 1985.

[13] H.C.Ombao, J.A.Raz,R.L.Strawderman, and R.Von Sachs, "A Simple Generalized Cross validation method of Span selection for periodogram smoothing”, Biometrika, vol.88,no.4,pp.1186-1192,2001.

[14] Thomas C.M.Lee, "A stabilized bandwidth selection method for kernel smoothing of the Periodogram" Signal processing 81 (2001) 419-430.

[15] PAYAM REFAEILZADEH, LEI TANG, HUAN LIU, “Cross-Validation” Arizona State University

[16] Prabhu Babu, Erik Gudmundson, and Petre Stoica, "Automatic Cepstrum-based Smoothing of the Periodogram via Cross-Validation”, EUSIPCO 2008, August 25-29 Switzerland.

[17] E.Gudmundson, N.Sandgren, and P.Stoica, "Automatic Smoothing of Periodograms" in Proceedings of the 2006 IEEE International Conference on Acoustics, Speech and Signal processing, Toulouse, France,2006.

[18] Y.Ephraim and M.Rahim, "On second-order statistics and linear estimation of cepstral coefficients", IEEE Trans.Speech Audio Processing, vol.7, no.2, pp.162-176, 1999.

[19] G.Schwarz, "Estimating the dimension of a model" The Annals of Statistics, vol.6, no.2, pp.461-464, Mar.1978.

[20] E.L. Lehmann, “Testing Statistical hypotheses”, John Wiley \& Sons, 1970.

[21] J.Rissanen, "Information and Complexity in statistical Modeling," Springer Verlag, 2007.

[22] P. Moulin, "Wavelet thresholding techniques for power spectrum estimation," IEEE Trans. Signal Processing, vol. 42, no. 11, pp. 3126-3136, 1994.

[23] H.-Y. Gao, "Choice of thresholds for wavelet shrinkage estimate of the spectrum," J. Time Series Anal., vol. 18, no. 3, pp. 231-251, 1997.

[24] A.T. Walden, D.B. Percival, and E.J. McCoy, "Spectrum estimation by wavelet thresholding of multitaper estimators," IEEE Trans. Signal Processing, vol. 46, no. 12, pp. 3153-3165, 1998.

[25] A.R. Ferreira da Silva, "Wavelet denoising with evolutionary algorithms," in Proc. Digital Sign., 2005, vol. 15, pp. 382-399. 
Signal \& Image Processing : An International Journal (SIPIJ) Vol.2, No.4, December 2011

\section{Authors}

M.VENKATANARAYANA was born on May $15^{\text {th }}, 1977$ at Gooty, Anatapur in A.P. He received his M.Tech in Electronics Instrumentation and Communication Systems from SVUCE, Tirupati, India Since then he is working as associate Professor in Department of ECE at KSRM College of Engg., Kadapa, India. He has 10 years experience of teaching undergraduate and post graduate students. $\mathrm{He}$ has published 08 research papers in National/International conferences and journals. His research interests are in the areas of signal processing, Spectral analysis, optimal filtering and Image processing.

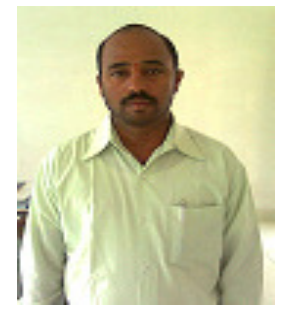

Dr.T.Jayachandra Prasad obtained his B.Tech in Electronics and Communication Engg., from JNTU College of Engineering, Anantapur-515002, and Master of Engineering degree in Applied Electronics from Coimbatore Institute of Technology, Coimbatore. He earned his Ph.D. Degree (Complex Signal Processing) in ECE from JNTUCE, Anantapur, India. Dr.T.Jayachandra Prasad worked in KSRM College of Engineering (KSRMCE), Kadapa, India from August 1984 to May 2006 in various positions such as Assistant professor, Associate professor and Professor and HOD. He worked as Head of ECE Dept. for 9 years at KSRMCE, Kadapa. He was instrumental for the establishment of various laboratories at KSRMCE. Later he joined in RGM College of Engineering and Technology, Nandyal, Kurnool (district), Andhra Pradesh (state), INDIA. Presently, he is the Principal of RGM College of Engineering and Technology, Nandyal. Dr.T.Jayachandra is having more than 25 years of experience and has more than 20 technical publications in International journals and National Journals. He is a life member of ISTE (India), Fellow of Institution of Engineers (Kolkata), Fellow of IETE, Member of MIEEE and life member of NAFEN. His areas of interest include Signal Processing and Image Processing.

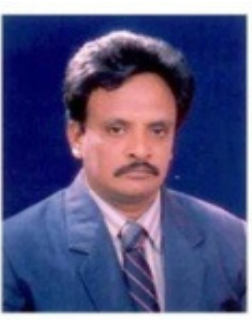

